nodule, presumed site of the tick bite accursed two months prior. She did not develop fever, arthralgias or other systemic symptoms. In order to exclude autoimmune diseases and infectious etiologies, we performed laboratory exams, such as anti-thyroid, antinuclear, anti-transglutaminase, TORCH and anti-Borrelia antibodies, resulted negative. A punch biopsy specimen from the scalp $(0.4 \times 0.3 \times 0.2 \mathrm{~cm})$ revealed fibrosis of the derma and the peripheric areas of pili-sebaceous annexes. The following month, we observed a rapid centrifugal progression to total alopecia. Thus, we decided to attempt therapy with topic corticosteroids followed by a progressive hair regrowth during the following four months.

Tick bite alopecia was first described in 1921. Since then, a few other cases have been reported in the international literature. The characteristic manifestation is a single zone of alopecia, often with a centrifugal spread, that appears 1-2 weeks after the tick removal. Sometimes, it can be associated with a central eschar, representing the site of tick bite. The nonscarring forms of alopecia manifest as 'moth-eaten' patches or, in alternative, as nodular or blood-crusted lesions. Clinically, patients may present with pain, pruritus or swelling. The precise mechanism for hair loss is not well understood but it is assumed to be caused by the host inflammatory response to tick saliva antigens. The result is the destruction of hair follicles or the alteration of the catagen/telogen phase. Histologic findings may show a heterogeneous inflammatory infiltrate and areas of fibrosis. The international literature does not report effective therapy for tick bite alopecia, while treatment with topic corticosteroids for alopecia areata is recognized. Prognosis is favourable with a complete hair regrowth usually within 3 months, although in some cases alopecia is reported to persist for 5 years.

\section{GP90 VACCINE INDUCED PERSISTENT SKIN REACTION WITH LOCALISED ACQUIRED HYPERTRICHOSIS}

${ }^{1}$ Susie van Robertson*, ${ }^{2}$ Geraldine Morrow. ${ }^{1}$ Mayo University Hospital, Castlebar, Ireland; ${ }^{2}$ Northbrook Clinic, Ranelagh, Ireland

\subsection{6/archdischild-2019-epa.155}

Background Vaccine induced persistently itchy granulomas are described in the setting of aluminium adsorbed vaccine preparations. ${ }^{1-2}$ Acquired localised hypertrichosis (ALH) has been attributed to inflammation yet rarely reported at vaccination sites $^{2}$. A Swedish longitudinal study estimated the incidence rate of vaccine induced granulomas as $0.8 \% \%^{3}$. $77-95 \%$ of those where contact allergic to aluminium with sensitivity to aluminium decreasing over time ${ }^{3}$. The granulomas are reported to resolve spontaneously over a number of years. ${ }^{3}$ The Meningococcal B 'Bexsero' vaccine is aluminium adsorbed and given by intramuscular injection.

Case We present the case of a 3 year old female with a vaccine induced persistent subcutaneous nodule and ALH. At age 12 months she received an intramuscular injection of Bexsero to her left anterolateral thigh. Within 2 weeks she developed a subcutaneous nodule at the injection site with an associated intensely localised pruritus causing her discomfort. Examination revealed a non tender subcutaneous nodule with an overlying patch of lichenified eczema with prominent growth of fine brown hair. Unfortunately, the pruritus was recalcitrant to treatment with occlusive dressings, emollients or application of $1 \%$ hydrocortisone cream. There was a good response however to topical Clobetasol but on ceasing application the pruritus flared immediately.

Her parents were reassured of the benign nature of the nodule and likelihood that a contact allergy to aluminium had developed. Caution with future aluminium exposure was recommended. They were advised that it may take up to a number of years, if at all, for the nodule to fully resolve and that studies into long term outcomes of the reaction are lacking. Nevertheless, their experience did not deter them from the national vaccination schedule. The patient has not experienced any further adverse reactions to vaccinations received.

Discussion Vaccine induced persistently pruritic subcutaneous nodules with ALH have been associated with aluminium adsorbed vaccines. The long term sequelae, if any, to this reaction is unknown. It is important for clinicians to recognise this reaction as a potential side effect so as to avoid unnecessary medical investigation.

\section{REFERENCES}

1. Bergfors $E$, Lundmark $K$, Nyström U. A child with a long-standing, intensely itching subcutaneous nodule on a thigh: an uncommon (?) reaction to commonly used vaccines. BMJ Case Rep. 2013.

2. Manchanda K, Mohanty S. Localized Hypertrichosis Following Vaccination in an Infant. Int J Trichology 2016.

3. Gente Lidholm A, Bergfors E, Inerot A, Trollfors B. Unexpected loss of contact allergy to aluminium induced by vaccine. Contact Dermat 2013.

\section{GP91 SCREENING FOR CYSTIC FIBROSIS RELATED LIVER DISEASE WITH ULTRASONOGRAPHY}

${ }^{1}$ Hannah Cooney*, ${ }^{1,2,3}$ Shivanthan Shanthikumar, ${ }^{1,2,3}$ Philip Robinson. ${ }^{1} T h e$ Royal Children's Hospital, Melbourne, Australia; '2Murdoch Children's Research Institute, Melbourne, Australia; ${ }^{3}$ University of Melbourne, Melbourne, Australia

10.1136/archdischild-2019-epa.156

Introduction Respiratory manifestations of cystic fibrosis (CF) have historically been the factor limiting prognosis. As there have been improvements in management of respiratory issues, increased screening and management of other manifestations of CF is required. CF related Liver Disease (CFLD) usually presents before or during adolescence and 5-10\% of patients develop clinically significant disease. Diagnosis of CFLD is defined by two of the following; abnormal clinical examination, persistent liver function test (LFT) derangement or ultrasonographic evidence of disease. Available clinical guidelines for infants with CF recommend screening for CFLD. The Royal Brompton Care of Children with CF guideline and the CF Trust Standards of Care suggest routine abdominal ultrasound (US) screening from age 5. In contrast, the NICE guidelines do not specify an age at which to commence screening. Given the high treatment burden for patients with $\mathrm{CF}$, we examined whether routine abdominal ultrasound screening for CFLD at age 5 leads to intervention or changes in clinical management.

Method We conducted a retrospective analysis of routine abdominal US screening conducted in CF patients at age 5, at the Royal Children's Hospital (Melbourne, Australia). The primary outcome measure was whether the US results led to a clinical intervention defined as treatment with ursodeoxycholic acid. Additional data collected included patient demographics, 
pancreatic and nutritional status, LFT result (normal, clinically insignificant, or clinically significant based on Royal Brompton Care of Children with CF guideline), referral to a hepatologist and timing of next US.

Results Between 2012 and 2017, 31 CF patients had a US at age 5 years. 22 patients $(71.0 \%)$ had no sonographic evidence of CFLD at time of first screening US. The remaining 9 patients $(29.0 \%)$ displayed only mild evidence of CFLD. One patient $(3.1 \%)$ was commenced on ursodeoxycholic acid. Two patients $(6.2 \%)$ were referred to a hepatolgoist. Follow up US was conducted an average of approximately 2 years following initial US. Mild clinically insignificant LFT derangement was common $(74.1 \%)$ but, all patients who had completely normal LFT results had no US evidence of disease.

Conclusions These results suggest that US conducted at age 5 rarely alters clinical management. Although US is a non-invasive investigation, CF patients already have a high treatment burden and given US at 5 years is highly unlikely to change management, it is unnecessary (especially in those with normal LFT's). Further investigation is needed to determine the optimal age to commence US screening for CFLD.

\section{GP92 THE EXPERIENCE OF A DAILY HOSPITAL WIDE OPERATIONAL HUDDLE AT TEMPLE STREET CHILDREN'S UNIVERSITY HOSPITAL}

Elaine Fitzgerald, Claire Fagan, Grainne Bauer, Sharon Ryan, Charlotte O'Dwyer, Sarah Maidment*, Clara Murtagh. CHI@Temple street, Dublin, Ireland

\subsection{6/archdischild-2019-epa.157}

The increasing complexity of healthcare delivery makes care co-ordination and maintaining safety a continuous challenge. Adverse events persist at a rate of $10.7 \%$ and half of such events being preventable (Stockwell et al. 2018)

The daily co-ordination of organisational needs results in a significant amount of information exchange across departments and individuals. This can affect the ability to plan and manage thus contributing to patient safety risk. Inter-dependant complex processes need a significant amount of information exchange and miscommunications could occur.

- What was the initiative taken?

A daily operational huddle to discuss key issues and plan ahead was piloted in 2014. The change was sustained and the huddle continuously improved and is now fully embedded.

- How was the change implemented?

Evidence from other organisations was reviewed. A format was designed and piloted amongst nursing staff initially. One representative from each department attended daily. Feedback and lessons learned were incorporated over time and the process expanded to include multiple departments. A pro forma whiteboard and huddle room was introduced in 2016.

- What problems were encountered with the process of change and how were these overcome?

Concerns regarding time to attend were initially expressed by frontline staff. Resolved by discussing benefits, visibility of teamwork in action through sharing resources and agreeing solutions. Additionally, it was agreed that attending the huddle reduced interruptions by phone calls.

A survey of huddle participants revealed the following results;

\begin{tabular}{|c|c|c|}
\hline & Question & Answer \\
\hline 1 & $\begin{array}{l}\text { In the last week I have been made aware of a risk via the } \\
\text { huddle }\end{array}$ & $52 \%$ agreed \\
\hline 2 & $\begin{array}{l}\text { Information about the outcome of past risks is provided at the } \\
\text { huddle }\end{array}$ & $64 \%$ agreed \\
\hline 3 & $\begin{array}{l}\text { I am comfortable sharing an incident or risk from my department } \\
\text { at the huddle }\end{array}$ & $86 \%$ Agreed \\
\hline 4 & The huddle is useful for me in my role & $95 \%$ Agreed \\
\hline 5 & I have learned new things at the huddle & $92 \%$ Agreed \\
\hline 6 & I am comfortable giving my report at the & $95 \%$ Agreed \\
\hline
\end{tabular}

Abstract GP92 Table 1 Percentage of huddles where key categories were discussed

\begin{tabular}{lll}
\hline Area & Total & $\%$ all huddles \\
\hline PEWS & 36 & $40 \%$ \\
Family Concerns & 12 & $13 \%$ \\
Stepped down from ICU & 47 & $52 \%$ \\
Patients with multiple teams involved & 9 & $10 \%$ \\
High risk therapies & 49 & $54 \%$ \\
Watchers & 37 & $41 \%$ \\
Good catch Identified & 4 & $4 \%$ \\
Clear Leader & 74 & $82 \%$ \\
\hline
\end{tabular}

- How did this improve patient/client care?

A daily forum now exists for planning, problem solving, identification of risks, sharing of lessons learned, sharing of resources and mass communication.

\section{GP93 EATING DISORDERS- A DESCRIPTIVE STUDY OF YOUNG PATIENTS WHO PRESENTED TO THE TALLAGHT UNIVERSITY HOSPITAL FOR ACUTE INTERVENTIONS AND MEDICAL STABILIZATION}

Nimantha Michael Gamage, Patricia Byrne*, Caroline McGrath. Liaison Child Psychiatry, Tallaght University Hospital, Dublin, Ireland

\subsection{6/archdischild-2019-epa.158}

Aim Anorexia Nervosa (AN) is a serious disorder with lifethreatening physical and psychological complications. Adolescents with AN have a standard mortality ratio 10 times of peers, primarily through physical complications. This study was carried out to investigate presentations of young patients under 16 years with restrictive eating disorders to Tallaght University Hospital, and evaluate standards of assessments and clinical practise compared to current best practice, the Junior MARSIPAN Guidelines (RCPsych, 2015).

Method A retrospective case study was conducted on all young patients (under 16) referred to the Liaison Child Psychiatry service with restrictive eating disorders between January 2014 and December 2018. Data from all available clinical records was examined. Baseline demographic variables, pathways of care, presenting symptoms and medical interventions were recorded. Clinical records were examined for evidence of recorded information regarding parameters of the JMARSIPAN risk assessment guidelines at admission and discharge. 